\title{
CMS Detector: Performance Results
}

\section{Redondo ${ }^{\star}$}

\section{CIEMAT}

Av. Compluense 40 Madrid 28040, Spain

E-mail: ignacio.redondo@ciemat.es

The Compact Muon Solenoid (CMS) detector is one of the two multipurpose experiments at the Large Hadron Collider (LHC). After an extremely successful first LHC run, CMS entered a phase of maintenance and upgrade in February 2013, when LHC stopped operation. This paper presents a brief selection of performance results obtained during the 2012 run, focusing on the objects that lead to the Higgs discovery measurements and putting some emphasis on pp collision event pileup dependencies, which will become relevant at the higher luminosity expected in the next LHC run.

The European Physical Society Conference on High Energy Physics

18-24 July, 2013

Stockholm, Sweden

\footnotetext{
* On behalf of the CMS Collaboration

† Speaker
} 


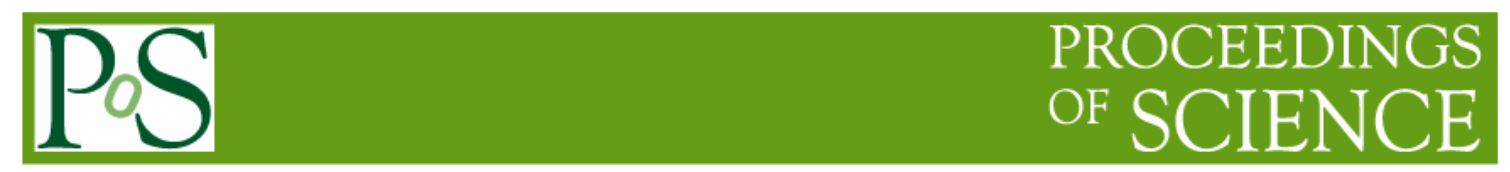




\section{CMS Detector Status}

The Compact Muon Solenoid (CMS) detector is designed to cope with the high occupancy and large energy of the collisions provided by the LHC. Being a classical collider detector with cylindrical geometry, its compactness and modular design, allows the flexibility of opening the detector for maintenance during long shutdowns. During the current shutdown (LS1), repairs were performed on all detector systems from the muon chambers to the innermost part (pixels), which were extracted and brought to surface to repair broken channels. Additionally, two forward muon discs are being installed in the forward direction completing the original CMS design. Further goals of LS1 are an upgrade of the tracker cooling system and an installation of a narrower beam pipe, heralding a future pixel upgrade. A detailed description of CMS can be found in [1]. The excellent performance of CMS is supported by the following (among others) quite unique characteristics:

- All Si tracker, largest of its kind, which provides the high granularity required to reconstruct heavy ion collisions or high pileup pp events.

- Calorimeters inside a large $3.8 \mathrm{~T}, 3 \mathrm{~m}$ radius solenoid.

- Hermetic up to 5.2 in rapidity. Lead Tungstate crystal based ECAL up to 3.

- Muon detectors, consisting of gas chambers, which provide redundant trigger and tracking over a large volume.

- A fast Level-1 programmable hardware trigger which filters events down to about $100 \mathrm{kHz}$, maximum speed at which the large $\sim 1 \mathrm{MB}$ event size is read out.

\section{CMS Operation during LHC Run I}

One of the major challenges of the LHC experiments is being able to reconstruct superposed collisions in the same $25 \mathrm{~ns}$ bunch crossing, denoted as pileup. Figure 1 shows the evolution of the maximum number of overlapping events during the first LHC run.

CMS peak interactions per crossing, pp

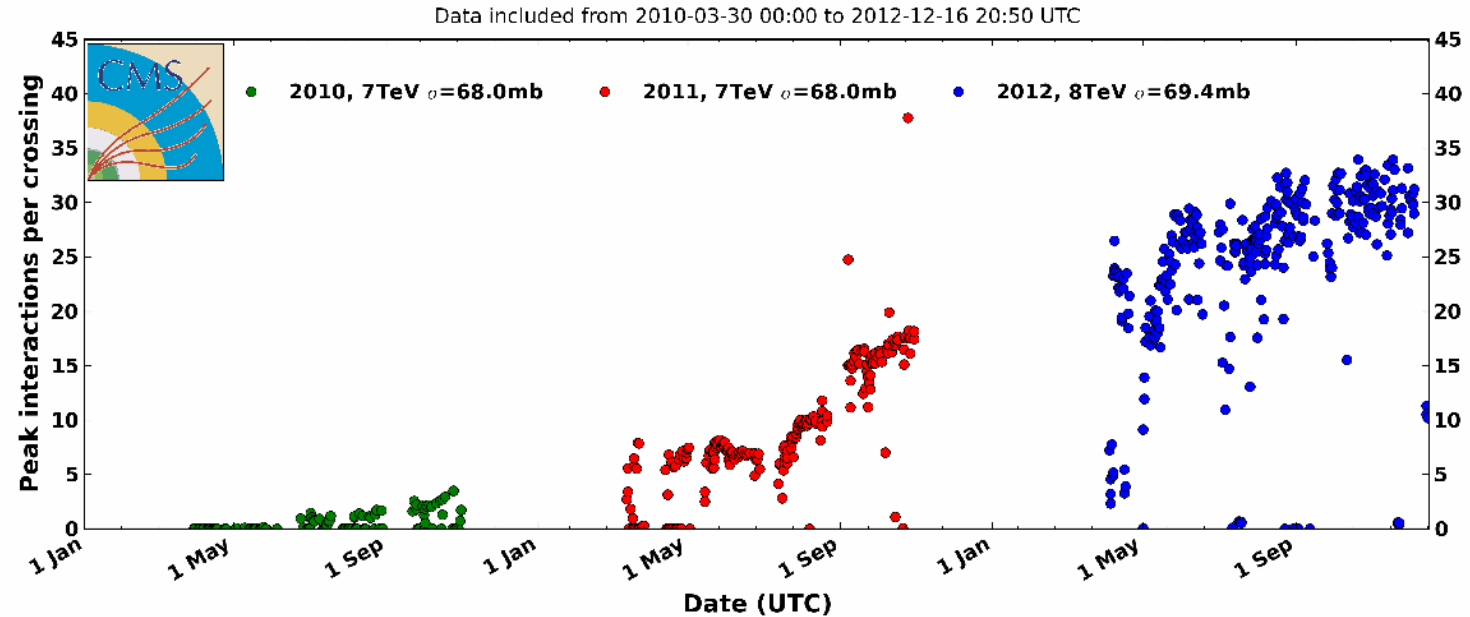

Figure 1: Peak number of interactions per crossing in CMS vs. date. 

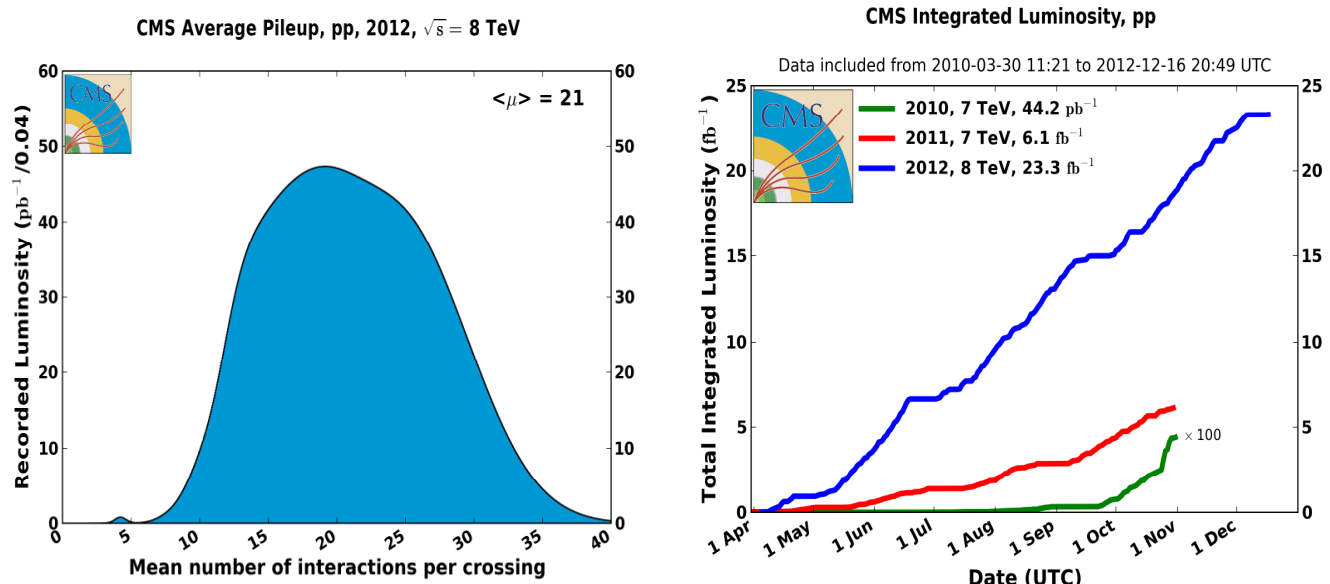

Figure 2: Distribution of the number of interactions per crossing of LHC beams in CMS during 2012 (left). Total integrated luminosity by CMS vs. date. (right).

Data taking conditions varied constantly as the accelerator increased its instantaneous luminosity. As a figure of merit of this increase, the trigger rate of muons in the barrel went from rate dominated by cosmics to (if no transverse momentum cut would be applied) saturate the $\mathrm{L} 1 \mathrm{maximum}$ rate of $100 \mathrm{kHz}$. Following this instantaneous luminosity growth, the mean number of interactions per crossing increased from 1 at the beginning of the run to 21 at the end (Figure 2 left). Most of the width of the distribution in 2012 comes from the instantaneous luminosity drop during a fill ( $\sim 12 \mathrm{~h})$. PU increase forced CMS to adapt to varying data taking conditions; these rang from firmware changes in several custom modules to changes in the trigger algorithms (trigger menus). In this area the strategy was to move offline algorithms (using particle flow reconstruction techniques) to the High Level Trigger (see Figure 3 left an example). Excellent data taking efficiency (90\% for data used in analysis) was achieved, not least thanks to a good deal of automation in the data taking control.

The healthy evolution of integrated luminosity shown in Figure 2 right allowed for a full scan of standard model processes as sensitivity to $\mathrm{O}(\mathrm{fb})$ cross sections was being approached, leading finally to the announcement of the discovery of a Higgs boson.
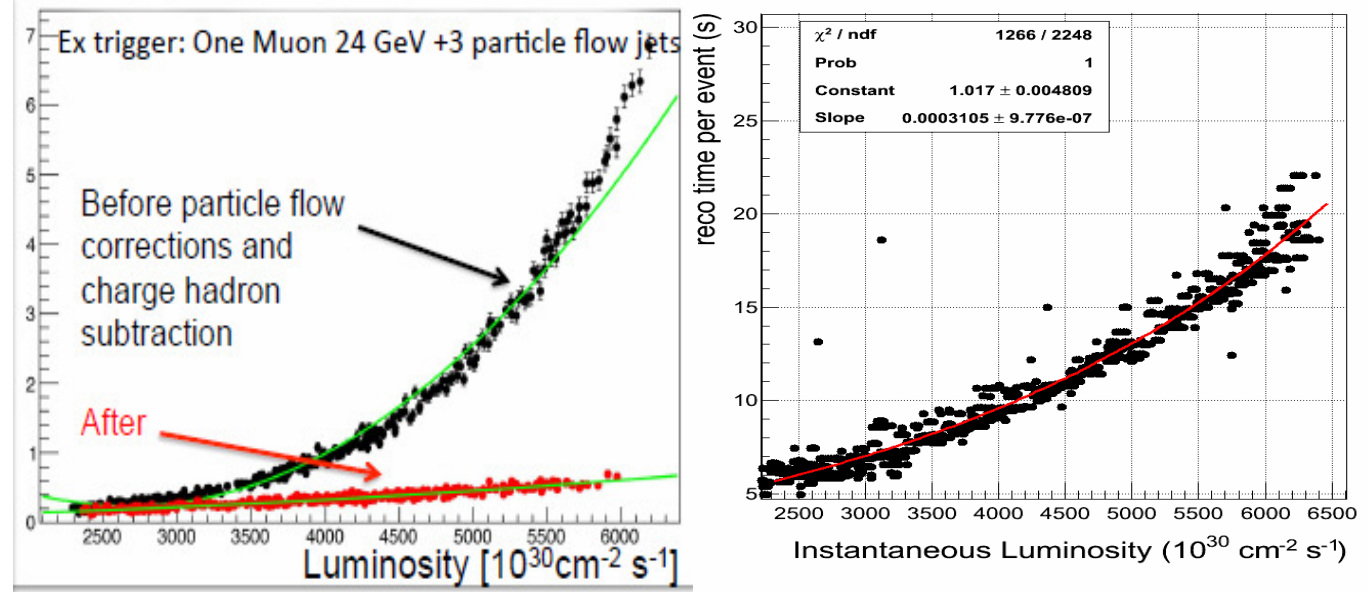

Figure 3: Trigger rate $(\mathrm{Hz})$ of an example High Level Trigger algorithm (left) and reconstruction time per event (s) (right) as a function of instantaneous luminosity $\left(10^{30} \mathrm{~cm}^{-2} \mathrm{~s}^{-1}\right)$. 


\section{Selected Performance Results}

Excellent performance from several systems under a mean event pileup of 20 interactions/crossing was required in order to discover a Higgs boson. Performance results regarding alignment [2], b-quark identification [3], and jet [4] performance have also been presented at this conference. The following focuses on a few key benchmarks that were crucial for the $\mathrm{H} \rightarrow 41$ and the $\mathrm{H} \rightarrow \gamma \gamma$ searches: i) massive computing resources; ii) tracking and vertexing iii) muon trigger and reconstruction; iv) electron trigger and reconstruction; v) photon trigger and identification.

\subsection{Computing and Data handling}

In addition to the large numbers of events (20 billion simulated events, 12 billion data events) and data volumes ( $>25$ PB moved to Tier-1s, 70 PB moved to Tier-2s) there is the strong increase of the event processing time with instantaneous luminosity, as shown in Figure 3 right. This result already includes improvements in the reconstruction code [5] that had to be implemented to cope with pileup during 2012 while maintaining the physics performance. Computing resources needed for next LHC run (all things equal) will increase by a factor of 10 , coming both from the non-linearity in Figure 3 right and the sheer larger number of events to be reconstructed.
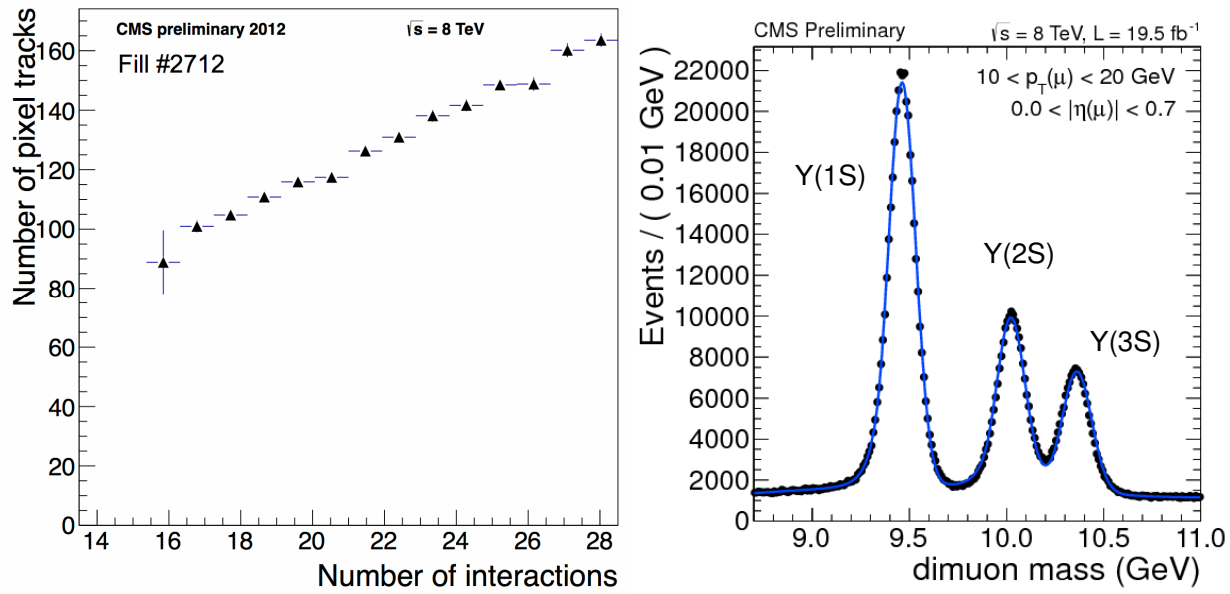

Figure 4: Number of pixel vertices as a function of the number of interactions(left). Dimuon mas in the $\mathrm{Y}$ resonance mass region (right).

\subsection{Tracking and Vertexing}

Tracking and vertexing are the backbone of the global event description. The number of pixel vertices (Figure 4 left) still showed a linear behaviour as a function of the number of interactions (reconstructed from the instantaneous luminosity as measured from the HF calorimeter in the forward region). A zoom in the dimuon mass distribution (Figure 4 right) in the Y mass region highlights the excellent tracking resolution.

\subsection{Muon Trigger and Reconstruction}

On the trigger side, the effort in 2012 was concentrated on trigger rate reduction. 
Figure 5 left shows the few percent drop in efficiency paid to achieve $50 \%$ trigger rate reduction in 2012 by means of: i) a tighter $\mathrm{p}_{\mathrm{T}}$ assignment in the endcap track finder; ii) improved barrel-endcap overlap track finding and iii) a better global muon merging of the several candidates available
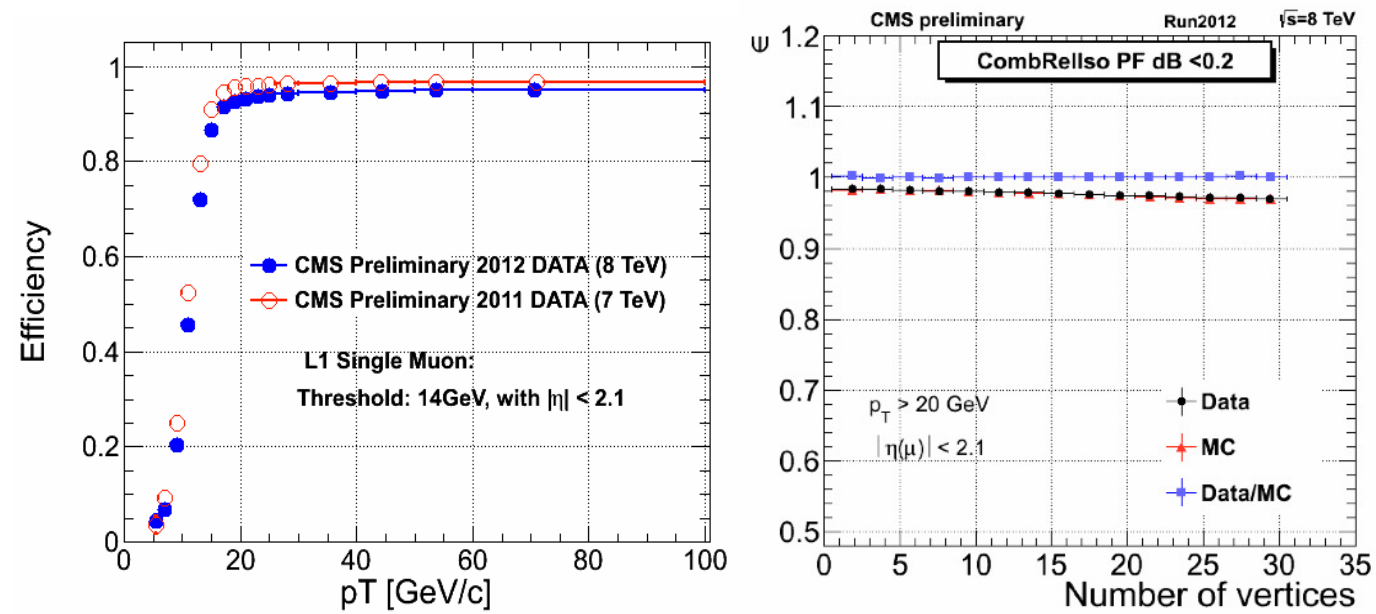

Figure 5: Left: L1 single muon trigger efficiency as a function of muon $\mathrm{p}_{\mathrm{T}}(\mathrm{GeV} / \mathrm{c})$ during 2011 (red circles) and 2012 (blue dots). Muon isolation efficiency as a function of the number of vertices (right).

The excellent muon $\mathrm{p}_{\mathrm{T}}$ reconstruction resolutions of $<1-2 \%$ in the barrel and $<6 \%$ in the endcap regions has been demonstrated early in the run by means of the reconstruction of all the dimuon resonances, a very popular plot that shows one advantage of hadron colliders: they provide an energy scan by design. It should be mentioned that the resolution is completely dominated by the strip tracker at momenta below $100 \mathrm{GeV} / \mathrm{c}(\Delta \mathrm{p} / \mathrm{p} \sim 1 \%$ @ $100 \mathrm{GeV} / \mathrm{c})$. Figure 5 right illustrates the rather flat behaviour of the muon isolation efficiency as a function of the number of vertices (a proxy for the number of interactions per crossing, see Figure 5 left) and the excellent description of the small decreasing trend by the simulation.
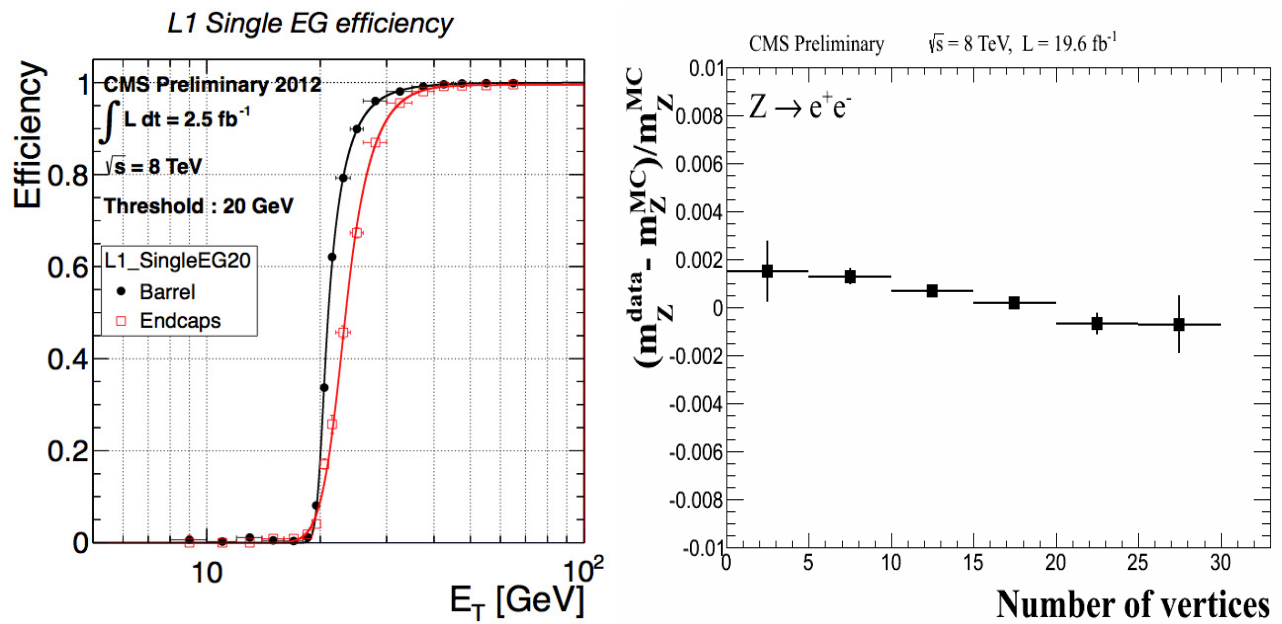

Figure 6: $\mathrm{L} 1$ single EGamma trigger efficiency vs object $\mathrm{E}_{\mathrm{T}}[\mathrm{GeV}]$ (right) for the barrel (black dots) and endcap (red circles) regions. Relative shift of the $\mathrm{Z}$ peak reconstructed from $\mathrm{e}^{+} \mathrm{e}^{-}$pairs as function of the number of vertices (right). 


\subsection{Electron Trigger and Reconstruction}

Figure 6 left displays the L1 trigger efficiency of electron candidates as a function of $E_{T}$ for both the encap and barrel regions. The implementation at L1 of corrections coming from laser monitoring during 2012 improved both the steepness of the threshold onset and the homogeneity between barrel and endcap.

Figure 6 right shows the flat behaviour of the Electromagnetic Calorimeter scale as a function of the number of vertices using the position of the $Z \rightarrow$ ee peak From the width of the peak, the resolution stability during 2012 run was measured to be better than $0.1 \%$. The scale was also studied using the $Z \rightarrow \mu \mu \gamma$ peak, which is a $\sim 99.6 \%$ pure source of photons, obtaining compatible results.

\subsection{Photon Identification}

The total photon identification scale factor is determined by the product of the scale factor calculated using a tag and probe technique and the scale factor of the electron veto. The electron veto scale factor is calculated from data and simulation by counting the number of passing and failing photons coming from the $\mathrm{Z} \rightarrow \mu \mu \gamma$ decay process. A CMS contribution on the preshower detector, which helps in photon identification, was presented at this conference [6].

\section{Conclusion}

CMS has achieved a superb performance during the first LHC run. LS1 activities are progressing well, recovering the already low number of bad channels and paving the way to upgrades (see related contributions at this conference [7]) aimed to increase the CMS experiment's capability under future high luminosity LHC conditions.

\section{References}

[1] The CMS Collaboration, The CMS experiment at the CERN LHC, JINST 03 (2008) S08004

[2] J. Duarte Campderros, Alignment of the CMS Muon System; N. Bartosik, Simultaneous alignment and Lorentz angle calibration in the CMS silicon tracker using Millepede II, these proceedings (EPS 2013).

[3] H. Kirschenmann, Jet performance in CMS; A. Schmidt CMS results on boosted-objects \& jet substructure, these proceedings (EPS 2013).

[4] I. Marchesini, Identification of b-quark Jets in the CMS experiment, these proceedings (EPS 2013).

[5] R. Castello, Data preparation for the Compact Muon Solenoid experiment, these proceedings (EPS 2013).

[6] C.P. Chang, Energy clustering, Calibration and Particle Identification with the CMS Preshower detector, these proceedings (EPS 2013).

[7] C. Fountas, Upgrade of the CMS Detector; K. Klein, Upgrade of the CMS Tracker; M. Abbrescia, Upgrade of the CMS Muon System; F. Cavallari, Upgrade of the CMS ECAL, these proceedings (EPS 2013). 